\title{
An ontology of sstronomical object types for the Virtual Observatory
}

\author{
Sebastian Derriere ${ }^{1}$, André Richard ${ }^{1,2}$ and Andrea Preite-Martinez ${ }^{1,2}$ \\ ${ }^{1}$ CDS, Observatoire astronomique de Strasbourg, UMR 7550, \\ 11 rue de l'Université, F-67000 Strasbourg, France \\ email: derriere@astro.u-strasbg.fr \\ ${ }^{2}$ INAF - Istituto di Astrofisica Spaziale e Fisica Cosmica, \\ Via del Fosso del Cavaliere 100, I-00133 Roma, Italy
}

The Semantic Web and ontologies are emerging technologies that enable advanced knowledge management and sharing. Their application to Astronomy can offer new ways of sharing information between astronomers, but also between machines or software components and allow inference engines to perform reasoning on an astronomical knowledge base. The first examples of astronomy-related ontologies are being developed in the european VOTech project.

We present the current status of an ontology describing knowledge about astronomical object types, originally based on the standardization of object types used in the SIMBAD database. We discuss the strategies that have been adopted for structuring this ontology, the problems that have been solved during the construction, and the links with current works in the Semantics working group of the IVOA.

We present applications of this ontology on several use cases:

- Support advanced resource queries in the VO Registries, by using datasets correspondence with ontology concepts. Figure 1 presents snapshots of this use case: the astronomer selects a starting concept from a scroll-list; the concept hierarchy is then recursively explored to find all more specific concepts having an associated registry 'Subject' keyword; the registry can then be queried based on these keywords.

- Validate multiple object classification in SIMBAD by checking the consistency of the types associated to the object's identifiers.

- Refine object classification in SIMBAD when adding new measurements or identifiers.

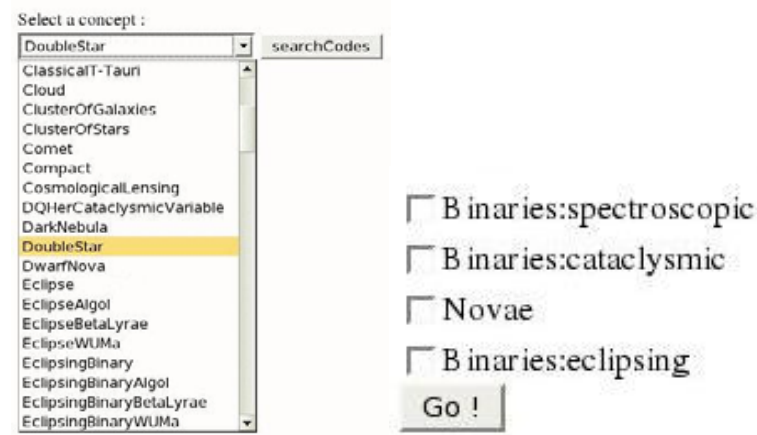

\begin{tabular}{|c|c|c|}
\hline AuthorityID & ResourceKey & Actions \\
\hline $\operatorname{CDS}$ & VizieA/J/A+A/289/871/aephe & View, Edit, XEdit \\
\hline $\operatorname{CDS}$ & VizieP/J/A+A/289/871/yyeri & View,Edit,XEdit \\
\hline $\operatorname{cDS}$ & VizieR/J/A+A/30Q/521/table1 & View, Edit, XEdit \\
\hline $\operatorname{CDS}$ & VizieR/J/A+A/312/93/table1 & View, Edit, XEdit \\
\hline CDS & VizieP/J/A+A/312/93/rets & Viow, Edit, XEdit \\
\hline $\operatorname{CDS}$ & VizieP/J/A+A/315/384/table3 & View,Edit,XEdit \\
\hline $\operatorname{CDS}$ & VizieP/J/A+A/319/481/table3 & View, Edit, XEdit \\
\hline $\cos$ & VizieP/J/A+A/319/867/table3a & View, Edit, XEdit \\
\hline CDS & VizieR/J/A+A/319/867/table3b & View,Edit,XEdit \\
\hline $\operatorname{CDS}$ & VizieP/J/A+A/324/137/table1 & Viow, Edit, XEdit \\
\hline
\end{tabular}

Figure 1. List of concepts related to astronomical objects in the ontology (left). Keywords corresponding to the selected concepts, and all more specific concepts (middle). List of relevant resources in the registry (right). 